\title{
Colour vision experimental studies in teaching of optometry
}

Maris Ozolinsh, Gatis Ikaunieks, Sergejs Fomins

Maris Ozolinsh, Gatis Ikaunieks, Sergejs Fomins, "Colour vision experimental studies in teaching of optometry," Proc. SPIE 9664, Ninth International Topical Meeting on Education and Training in Optics and Photonics, 96640I (24 October 2005); doi: 10.1117/12.2207734

SDIE Event: Ninth International Topical Meeting on Education and Training in Optics and Photonics, 2005, Marseille, France 
Ref ETOP070

\title{
Colour vision experimental studies in teaching of optometry
}

\author{
Maris Ozolinsh, Gatis Ikaunieks and Sergejs Fomins \\ Department of Optometry and Vision Science, University of Latvia, 8 Kengaraga Str., LV0- \\ 1063 Riga, LATVIA , ozoma@latnet.Iv
}

\begin{abstract}
s
Following aspects related to human colour vision are included in experimental lessons for optometry students of University of Latvia. Characteristics of coloured stimuli (emitting and reflective), determination their coordinates in different colour spaces. Objective characteristics of transmitting of colour stimuli through the optical system of eye together with various types of appliances (lenses, prisms, Fresnel prisms). Psychophysical determination of mono- and polychromatic stimuli perception taking into account physiology of eye, retinal colour photoreceptor topography and spectral sensitivity, spatial and temporal characteristics of retinal receptive fields. Ergonomics of visual perception, influence of illumination and glare effects, testing of colour vision deficiencies.
\end{abstract}

\section{Key words}

optometry, colour vision, colour gamut, visual perception, visual acuity, flicker fusion, heterochromatic flicker photometry

\section{Summary.}

Duties of optometrists as a specific and regulated profession nowadays are growing rapidly within the quality of technical means and clinical patient care, within increasing of visual stress intensity, and trends to higher patient visual comfort. Currently optometry teaching in different countries still is different instead of trends to obtain the coherence in education and expertise of optometrists at least within the European Union countries. Latvia has almost 15 year experience in optometrist teaching at bachelor and master levels following the curriculum similar and close to the curriculum in Great Britain, Ireland, Norway. Present report relates to the experimental studies of colour vision provided within training of optometrists in University of Latvia.

Knowledge of the following aspects related to human colour vision is included in practical experimental lessons for students going to be optometrists or vision scientists.

1. Characteristics of coloured stimuli (emitting and reflective ones), determination their coordinates in different colour spaces, generation of analog and digital colour stimuli, characteristics of different kind of digital stimuli output together with possibilities and requirements of its colour and intensity calibration.

2. Objective characteristics of transmitting of different colour stimuli through the optical system of an eye together with various types of eye appliances (lenses, prisms, Fresnel prisms, etc.) limiting the resolution of a real as compared with an "ideal" eye, impact of diffraction and eye aberrations on the resolving power of the eye, differences in the light scattering of different wavelength affecting the perception of monochromatic or polychromatic stimuli.

3. Visual perception psychophysical determination of mono- and polychromatic stimuli taking into account physiology of the eye, retinal colour photoreceptor topography and spectral sensitivity, spatial and temporal characteristics of retinal receptive fields.

4. Ergonomics of visual perception, influence of illumination and glare effects on the colour stimuli recognition, testing of colour vision deficiencies. 
The report presents and demonstrates a number of techniques used in laboratory lessons devoted to obtain knowledge on the previously mentioned colour vision characteristics (such as "Determination of colour display spectral emittance, colour gamut, gamma-function and display calibration", "Determination of reflective surface colour coordinates", "Optical transfer function determination (a model eye together with optical appliances: lenses, progressive lenses, Fresnel prisms)", "Light scattering of different wavelength emission in a cataract eye model", "Heterochromatic flicker photometry", "Colour stimuli visual acuity", "Polychromatic stimuli colour contrast sensitivity".

To determine emitting and reflective stimuli spectral characteristics, we used in the laboratory work a fibre "Ocean Optics" spectrometer supplied with a small integrating sphere. Normally the colour coordinates and the colour gamut of the display emission are determined in CIE XYZ and $L^{*} a^{*} b^{*}$ colour spaces. To perform display calibration procedure we used "Spider2Pro" photometer with software allowing us to rebuild the gamma function in the most proper way in all specific tasks, where the display is used to present visual stimuli.

Optical properties of "model eye + appliance" system are determined using a three colour RGB laser (CNITRA, wavelengths $-670 \mathrm{~nm}, 532 \mathrm{~nm}, 473 \mathrm{~nm}$ ) as well from the point spread function using a double path system as from the increase of the beam divergence.

In model experiments light scattering is induced using, firstly, a PDLC (polymer diffused liquid crystal) cell with controllable light scattering by applying different AC voltage. Secondly step-by-step light scattering is obtained using obstacles performed on composites of MMA (methyl methacrylate) glue with dispersed small glass microparticles. The refractive indices of PMMA $n=1.44$ and glass $n=1.5$ are close to the indices of the clear human eye lens and for cataract protein formation. Changing the concentration of the glass particles and the thickness of the composite layer it possible to adjust the light scattering level to different stages of the cataract development. Light scattering depends strongly on the wavelength and such obstacles are used in a number of laboratory works to study the effect of scattering on perception of different colour stimuli. Aberrations of a real human eye are determined using

"MultiSpot 250"

aberrometer.

Psychophysically the visual performance is studied in two ways using visual acuity letter (e.g., Landolt C) charts build of non-isoluminant stimuli with different colour contrast (white-blue, white-red, white-green, etc.), or quasimonochromatic (red, green, blue) stimuli of different intensity contrast. Secondly, the Gabor gratings are used for contrast sensitivity measurements using

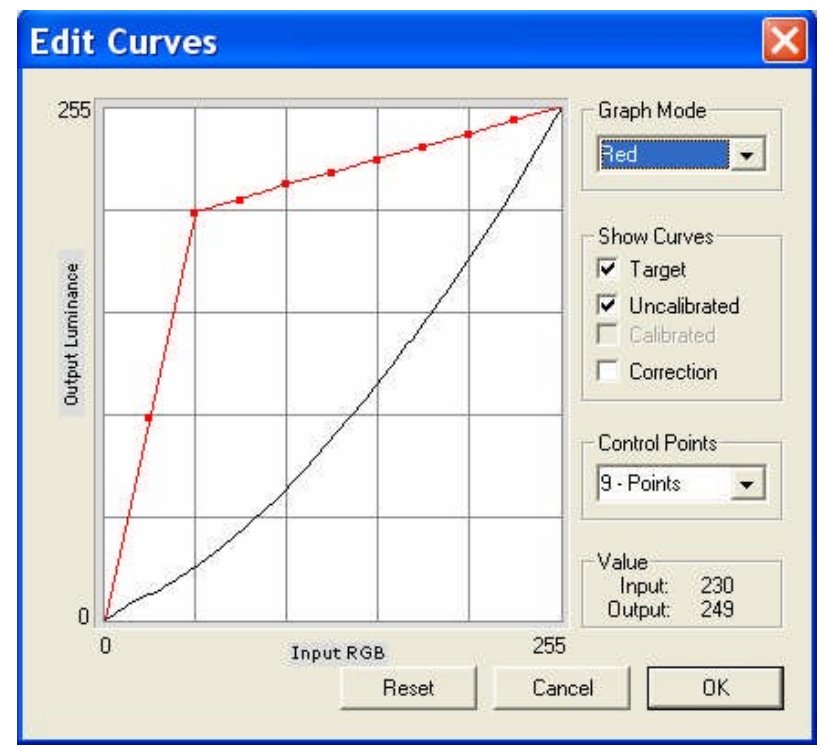

Fig. 1. Display luminance curve calibration using "Spider2 Pro" sensor. Solid line - initial Input/Output characteristics (luminance $=R_{G B}{ }^{g}$ with $\mathbf{g}=2$ ), line with dots - corrected Input/Output characteristics allowing obtain more gray scale levels for RGB range [50,2555], equivalent to $10 \mathrm{bit} / \mathrm{channel}$ videocard output. 
quasimonochromatic sinusoidal intensity modulated stimuli or similar structure isoluminant grating stimuli with colour contrast (opponent colours red-green, blue-yellow). These stimuli are generated on the PC screen and quasimonochromaticity of stimulus, e.g. "blue", actually means relatively broad emission of the blue CRT screen phosphors. The human vision is very sensitive to contrast changes (contrast sensitivity $\mathrm{CS}>100, \mathrm{CS}=$ $1 / C=($ Lmax + Lmin $) /($ Lmax-Lmin $)$, where C- Michelson contrast, Lmax and Lmin - stimuli maxima and minima luminance). Thus to build

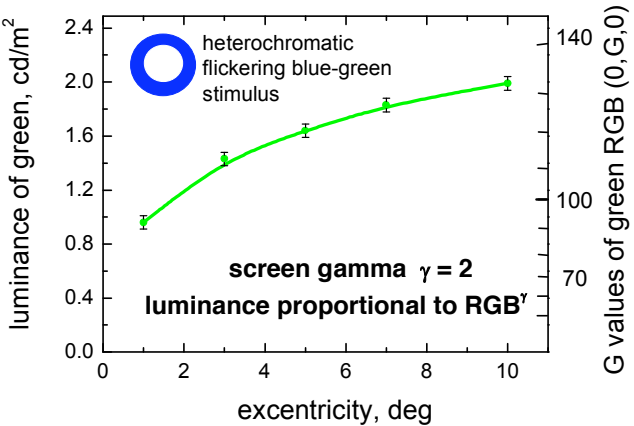

Fig.2. Heterochromatic flicker isoluminance point determination at different positions on human retina (excentricity in degrees from the central vision area) for CRT display blue $\operatorname{RGB}(0,0,255)$ with screen luminance $L=1.35 \mathrm{~cd} / \mathrm{m}^{2}$ and display green $\operatorname{RGB}(0, G, 0)$.

such stimuli on the PC display using 24bit RGB colour depth, the appropriate display Input/Output curve $L=L(R G B)$ providing fine contrast within large changes of $R G B$ values should be build by means of the "Spider2Pro" type photometer software (Fig.1).

Every man has his own colour sensitivity curve. To ensure the isoluminance of stimuli the relative sensitivity corresponding to three $R, G$ and $B$ phosphor emission should be determined using the heterochromatic flicker photometry. To do that for R and $\mathrm{G}$ phosphor emission two stimuli - red and green are periodically interchanged on the screen with frequency ca. $10-30 \mathrm{~Hz}$. At some $\mathrm{R}$ and $\mathrm{G}$ stimuli ratio value $\mathrm{R} / \mathrm{G}$, flickering of the stimuli is minimal or is disappearing completely, and the person doing experiments can assume the corresponding stimuli as isoluminant ones.

Also for humans with no colour vision deficiency the relative R/G sensitivity varies due to different $R$ and $G$ cone distribution within retina area. "Blue" short wavelength sensitive $S$ cones are practically absent in the fovea - the central vision area of retina has very low $S$ cone density.

Thus the relative sensitivity of $\mathrm{M}$ and $\mathrm{S}$ cones depends on the excentricity - on angular distance from the retina centre. In laboratory work, using as heterochromatic stimuli annulus with different inner and outer radii students determine their relative sensitivity of middle and short wavelength emission (data shown in Fig.2). 